\title{
DETERMINAÇÃO DE RESÍDUOS DE AGROTÓXICOS ORGANOCLORADOS EM HORTALIÇAS *
}

\author{
MARIA TERESA PLATA OVIEDO ** \\ MARIA CECÍLIA DE FIGUEIREDO TOLEDO *** \\ EDUARDO VICENTE
}

\begin{abstract}
Foram analisados resíduos de Clorotalonil, Aldrin, Dieldrin, Heptacloro, Heptacloro epóxido, Endosulfan alfa, Endosulfan beta e Sulfato de Endosulfan nas seguintes hortaliças: alface, acelga, chicória, repolho, tomate, chuchu, batata, cenoura, mandioca e mandioquinha. As amostras foram coletadas no restaurante da Universidade Estadual de Campinas, no período de abril/1998 a abril/ 2001 e nas Centrais de Abastecimento de Campinas, no período de abril a agosto/2001. Os resíduos dos agrotóxicos organoclorados foram determinados por cromatografia a gás com detector de captura de elétrons (CG-DCE- $\left.{ }^{63} \mathrm{Ni}\right)$. Os limites de detecção (LD) encontrados para Clorotalonil, Aldrin, Dieldrin, Heptacloro, Heptacloro epóxido, Endosulfan alfa, Endosulfan beta e Sulfato de Endosulfan foram 0,0006, 0,0006, 0,0008, 0,0006, 0,0007, $0,0008,0,0009$ e $0,0016 \mathrm{mg} / \mathrm{kg}$, e os limites de quantificação $0,002,0,002,0,003,0,002,0,003,0,003$, 0,003 e $0,005 \mathrm{mg} / \mathrm{kg}$, respectivamente. A recuperação média dos agrotóxicos analisados variou de 76,0 a $97,0 \%$ em repolho, de 90,0 a $103,0 \%$ em tomate e de 81,0 a $106,0 \%$ em batata. Em todas as amostras de hortaliças analisadas $(n=151)$, os níveis residuais de agrotóxicos apresentaram-se abaixo dos limites de detecção (LD) do método validado.
\end{abstract}

PALAVRAS-CHAVE: RESÍDUOS; AGROTÓXICOS-ORGANOCLORADOS; HORTALIÇAS.

\section{INTRODUÇÃO}

Os agrotóxicos são produtos químicos destinados a prevenir ou controlar pragas, incluindo vetores de doenças que causem prejuízo na

* Parte da Tese de Doutorado do primeiro autor.

** Doutora em Ciência de Alimentos, Faculdade de Engenharia de Alimentos (FEA), Universidade Estadual de Campinas (UNICAMP), Campinas, SP. (e-mail:matepo@hotmail.com).

*** Pra. Dra Titular de Toxicologia de Alimentos, Departamento de Ciência de Alimentos, FEA, UNICAMP, Campinas, SP. (e-mail:macecil@fea.unicamp.br).

**** Pesquisador Científico, Instituto de Tecnologia de Alimentos (ITAL), Campinas, SP. (e-mail: evicente@ital.org.br). 
produção, armazenamento, transporte e comercialização dos alimentos. Esses compostos representam grupo polêmico de substâncias. Se por um lado contribuem com o aumento da produção agrícola, por outro podem contaminar os alimentos e o ambiente caso não sejam respeitadas as boas práticas agrícolas. Desta forma, para garantir à população produtos que não representem risco à saúde é de grande importância o monitoramento de resíduos de agrotóxicos em alimentos disponíveis no comércio.

Embora a literatura consultada indique a ausência ou presença em níveis bastante baixos de agrotóxicos organoclorados em algumas hortaliças (UNGARO et al. 1987; ZANDONÁ e ZAPPIA, 1993; BARRETO et al. 1996), o conhecimento de suas propriedades tóxicas, seu caráter acumulativo e a possibilidade de venda ilegal exigem monitoramento contínuo.

A aplicação do fungicida Clorotalonil é autorizada em partes aéreas de culturas de amendoim, arroz, banana, batata, berinjela, café, cenoura, cebola, citros, feijão, fumo, maçã, mamão, melão, melancia, pepino, pimentão, soja, tomate, trigo, uva, seringueira, gramados e plantas ornamentais (ANVISA, 2002a). Os inseticidas Aldrin, Dieldrin, Heptacloro, Heptacloro epóxido, Endosulfan alfa, Endosulfan beta e Sulfato de Endosulfan foram proibidos pela Legislação Brasileira para uso na agropecuária pela Portaria no 329/85 (BRASIL, 1985). Excepcionalmente, o Endosulfan tem o seu emprego autorizado para aplicação em partes aéreas nas culturas de algodão, cacau, café, soja e cana-de-açúcar (ANVISA, 2002b). Resíduos de organoclorados podem eventualmente estar presentes em outras culturas, devido à aplicação indevida ou contaminação através do meio ambiente.

O objetivo desta pesquisa foi determinar os resíduos do fungicida Clorotalonil e dos inseticidas Aldrin, Dieldrin, Heptacloro, Heptacloro epóxido, Endosulfan alfa, Endosulfan beta e Sulfato de Endosulfan em hortaliças servidas nos restaurantes da Universidade Estadual de Campinas (UNICAMP) e comercializadas nas Centrais de Abastecimento de Campinas (CEASA).

\section{MATERIAL E MÉTODOS}

\subsection{AMOSTRAS, COLETA E ARMAZENAMENTO}

Foram coletadas no restaurante da UNICAMP amostras de $2 \mathrm{~kg}$ das seguintes hortaliças in natura: alface, acelga, chicória, repolho, tomate e mandioquinha, além de amostras de $5 \mathrm{~kg}$ de chuchu, batata, 
cenoura e mandioca, cuja procedência foi investigada. A quantidade de amostras coletadas, no período de abril/1998 a abril de 2001, obedeceu critérios adotados por YOKOMIZO et al. (1984) para análise de resíduos de agrotóxicos em vegetais in natura. As hortaliças foram acondicionadas em sacos de polietileno e transportadas em caixas de isopor com gelo, devidamente rotuladas e levadas ao laboratório de análises. Após homogeneização, em multiprocessador de alimentos (Walita), as amostras foram acondicionadas em frascos de vidro de $500 \mathrm{~mL}$ fechados com tampas envolvidas com papel de alumínio e armazenadas em congelador a $-18 \stackrel{\circ}{\circ} \pm 2 \stackrel{\circ}{ } \mathrm{C}$ até o momento da análise (realizada em duplicata).

Foram também analisadas hortaliças comercializadas nas Centrais de Abastecimento de Campinas no período de abril a agosto de 2001 ( 5 coletas mensais consecutivas). O procedimento adotado na coleta das amostras e posterior tratamento foi semelhante ao utilizado para as amostras da UNICAMP.

\subsection{PADRÕES E REAGENTES}

Foram preparados, em acetona grau pesticida (Mallinckrodt), soluções contendo $200 \mathrm{mg} / \mathrm{L}$ dos padrões de Clorotalonil com grau de pureza de 99,60\%, cedido pela Zeneca do Brasil Ltda, Aldrin ( $n^{\circ}$ 49000U-1), Dieldrin (no 49024-1), Heptacloro (no 749041-1), Heptacloro epóxido (n-4-9042) (Supelco, USA, todos com pureza de 99\%), Endosulfan alfa (no05261800ZB99005), Endosulfan beta (no 05261900ZB99003) e Sulfato de Endosulfan ( $n^{\circ}$ 05132700ZB98003) fornecidos pela Agrevo Hoechst do Brasil, com 99,80\%, 99,30\% e 97,50\% de pureza, respectivamente. A partir destas soluções foram obtidas, por diluição em hexano (p.r. Mallinckrodt), soluções padrão contendo 2,0, 1,5, 1,0, 0,5 e $0,2 \mathrm{mg} / \mathrm{L}$ dos agrotóxicos. Os reagentes utilizados foram $\mathrm{Na}_{2} \mathrm{SO}_{4} \mathrm{NaCl}$ e florisil (p.r. Mallinckrodt), tratados previamente em mufla durante 6 horas a $600{ }^{\circ} \mathrm{C}$, peneirados, guardados em frasco âmbar e conservados em dessecador com sílica gel até o momento da análise.

\subsection{MÉTODOS DE ANÁLISES}

A metodologia utilizada consistiu de extração e partição líquidolíquido, conforme o método modificado de LUKE et al. (1975), e limpeza das amostras, realizada em coluna de florisil, de acordo com MILLS et al. (1972). 


\subsubsection{Método modificado de LUKE et al. (1975)}

\section{Extração e purificação por partição líquido-líquido}

Aproximadamente 50 gramas de amostra, previamente homogeneizadas durante 30 segundos, foram extraídas com $150 \mathrm{~mL}$ de acetona p. r. e agitadas por 1 hora. Filtrou-se o extrato em funil Büchner com papel de filtro Whatman $n^{\circ} 41$ (tratado com acetona p.a. em Soxhlet durante 8 horas), o qual foi recolhido em kitasato acoplado à bomba de vácuo. Tranferiu-se o filtrado para balão volumétrico de $200 \mathrm{~mL}$, sendo o volume completado com acetona p.r. Uma alíquota de $50 \mathrm{~mL}$ do extrato foi transferida para funil de separação de $500 \mathrm{~mL}$ sendo acrescentados $100 \mathrm{~mL}$ de mistura hexano:diclorometano (grau para resíduo 50:50 v/v), tendo-se o cuidado de lavar uma vez o kitasato com a mistura de solventes. A seguir, foram adicionados $4 \mathrm{~g}$ de $\mathrm{NaCl}$ (tratado previamente em mufla) diluídos em $40 \mathrm{~mL}$ de água bidestilada, sendo a mistura agitada vigorosamente durante 5 minutos e então deixada em repouso para separação das fases orgânica e aquosa. Transferiu-se a fase aquosa para funil de separação de $500 \mathrm{~mL}$, sendo adicionados $50 \mathrm{~mL}$ de diclorometano p.r. A solução foi então agitada vigorosamente por 5 minutos e deixada decantar por 5 minutos até nova separação de fases. A adição foi repetida por duas vezes. As fases orgânicas foram combinadas e filtradas através de papel de filtro Framex $-15 \mathrm{~cm}$ com $5 \mathrm{~g}$ de $\mathrm{Na}_{2} \mathrm{SO}_{4}$ (tratado previamente em mufla a $600{ }^{\circ} \mathrm{C}$ durante 6 horas), utilizando-se funil como suporte. O filtrado foi recolhido em balão de evaporação fundo redondo de $500 \mathrm{~mL}$, concentrado até quase a secura em rotavapor com banho no máximo a $40{ }^{\circ} \mathrm{C}$ e seco com fluxo de nitrogênio. Foram adicionados $10 \mathrm{~mL}$ de hexano p.r. ao balão de evaporação.

\subsubsection{Limpeza do extrato em coluna cromatografica (MILLS et al. 1972)}

Preparou-se coluna cromatográfica de $2 \mathrm{~cm}$ de diâmetro interno e $30 \mathrm{~cm}$ de comprimento, empacotada com $10 \mathrm{~g}$ de florisil p.r. 100-120 mesh (tratado previamente em mufla a $600^{\circ} \mathrm{C}$ durante 6 horas). Adicionouse no topo da coluna $1 / 2 \mathrm{~cm}$ de $\mathrm{Na2SO} 4$ sendo a mesma eluída com $50 \mathrm{~mL}$ de hexano. Sem deixar a coluna secar transferiu-se, com pipeta de Pasteur, o extrato diluído em $30 \mathrm{~mL}$ do eluente I (hexano:diclorometano 99:1 v:v) para dentro da coluna com florisil, tendo-se o cuidado de lavar o balão de $500 \mathrm{~mL}$ com mais $70 \mathrm{~mL}$ do mesmo eluente. Passou-se 0 
eluente pela coluna e recolheu-se o eluato em balão volumétrico de $250 \mathrm{~mL}$. A seguir, foram passados pela coluna $100 \mathrm{~mL}$ do eluente II (hexano: diclorometano 80:20 v:v), seguidos de $100 \mathrm{~mL}$ do eluente III (hexano:diclorometano:acetonitrila 49,25:50,0:0,75 v:v). Os eluatos foram recolhidos separadamente em balões volumétricos de $250 \mathrm{~mL}$. Após concentração em rotavapor, no máximo a $40^{\circ} \mathrm{C}$ até quase à secura, e secagem com fluxo de nitrogênio, o extrato foi diluído com $10 \mathrm{~mL}$ de hexano. Foram injetados em cromatógrafo a gás $3 \mu \mathrm{L}$ do extrato diluído.

\subsubsection{Análise cromatográfica}

Utilizou-se cromatógrafo a gás VARIAN, modelo 3400, equipado com detector por captura de elétrons (CG-DCE-63 $\mathrm{Ni}$ ), coluna megabore DB-5 (30 m de comprimento, 0,53 mm de diâmetro interno e 1,5 $\mu \mathrm{m}$ de filme, fase estacionária $5 \%$ de fenil metil polissiloxano). Utilizou-se como processador de dados a Workstation 4,51, monitor e impressora. Foram injetados $3 \mu \mathrm{L}$ da amostra no modo on-column, com programação de temperatura em $170{ }^{\circ} \mathrm{C}$ (1 minuto), primeiro gradiente de $5^{\circ} \mathrm{C} / \mathrm{min}$ até $250 \stackrel{\circ}{ } \mathrm{C}$ (6 minutos), gás de arraste nitrogênio em $40 \mathrm{~mL} / \mathrm{min}$, temperatura do injetor em $240{ }^{\circ} \mathrm{C}$ e temperatura do detector em $300^{\circ} \mathrm{C}$. O tempo de duração de cada análise cromatográfica foi de 21 minutos. Os tempos de retenção absolutos dos padrões injetados foram: Clorotalonil 7,46 min, Heptacloro 8,85 min, Aldrin 9,94 min, Heptacloro epóxido 11,15 min, Endosulfan alfa 12,34 min, Dieldrin 13,19 min, Endosulfan beta 14,19 min e Sulfato de endosulfan 15,62 min.

A identificação dos organoclorados foi feita pela comparação dos tempos de retenção absolutos dos picos obtidos nas amostras com os respectivos padrões analíticos, e a quantificação pelo método de padronização externa. Curvas padrão analíticas foram construídas relacionando a área do pico versus concentração $(\mathrm{mg} / \mathrm{L})$ da mistura de soluções padrões de Clorotalonil, Aldrin, Dieldrin, Heptacloro, Heptacloro epóxido, Endosulfan alfa, Endosulfan beta e Sulfato de endosulfan, preparadas em hexano nas seguintes concentrações: 0,$01 ; 0,02 ; 0,03$; 0,04 e $0,05 \mathrm{mg} / \mathrm{L}$. Os coeficientes de correlação foram 0,9996, 0,9998, 0,9997, 0,9990, 0,9991, 0,9993, 0,9992 e 0,9994, respectivamente.

\subsubsection{Confirmação da identidade}

Cromatografia a gás com detector de captura de elétrons (CG-DCE $\left.{ }^{63} \mathrm{Ni}\right)$ 
Os testes para confirmação de identidade dos agrotóxicos organoclorados foram realizados no mesmo cromatógrafo a gás VARIAN3400 , acoplado ao detector por captura de elétrons. As condições cromatográficas utilizadas foram: coluna megabore VA-608 (30 m de comprimento $\times 0,53 \mathrm{~mm}$ de diâmetro interno $\times 0,83 \mu \mathrm{m}$ de filme). Programação de temperatura em $230^{\circ} \mathrm{C}$ (1 minuto), primeiro gradiente de $5 \stackrel{\circ}{\circ} / \mathrm{min}$ até $250 \stackrel{\circ}{\circ}$ (10 minutos), gás de arraste nitrogênio em $30 \mathrm{~mL} / \mathrm{min}$, temperatura do injetor $240^{\circ} \mathrm{C}$ e temperatura do detector em $300 \stackrel{\circ}{ } \mathrm{C}$. Inicialmente foram injetadas alíquotas de $3 \mu \mathrm{L}$ da mistura de padrões de interesse na concentração de $0,01 \mathrm{mg} / \mathrm{L}$ e em seguida, as amostras foram injetadas. O tempo de duração de cada corrida cromatográfica foi de 22,60 minutos. Os tempos de retenção absolutos dos padrões foram: Heptacloro 10,19 min, Aldrin 10,84 min, Heptacloro epóxido 11,97 min, Endosulfan alfa 12,73 min, Dieldrin 13,48 min, Endosulfan beta 15,15 min e Sulfato de endosulfan 16,97 min.

Cromatografía a gás acoplada ao espectrômetro de massas (CG-EM)

A confirmação da identidade dos agrotóxicos organoclorados foi realizada também por CG-EM, usando-se cromatógrafo a gás modelo HP 6890, amostrador automático HP 7683 acoplado a detector de massas HP 5973, operando com fonte de elétrons de 70 eV. Utilizou-se coluna capilar de sílica fundida HP5-MS (30 m de comprimento, 0,25 mm de diâmetro interno, 0,25 $\mu \mathrm{m}$ de filme, com $5 \%$ de difenil e $95 \%$ de dimetil polissiloxano), hélio como gás de arraste e vazão constante de $1,0 \mathrm{~mL} /$ min. As injeções foram feitas no injetor de temperatura programável (PTV), operando no modo splitless, mantido a $70^{\circ} \mathrm{C}$ por 0,1 minuto e programado até $250 \stackrel{\circ}{\circ} \mathrm{com}$ uma razão de $500^{\circ} \mathrm{C} / \mathrm{min}$, permanecendo nessa temperatura por 5 minutos. Após 5 minutos da injeção, a válvula de purga do injetor foi aberta por 1 minuto, com vazão de $0,1 \mu \mathrm{L}$ a $100 \mathrm{~mL} / \mathrm{min}$. $O$ volume injetado foi de $1 \mu \mathrm{L}$. Manteve-se a temperatura da coluna em $70^{\circ} \mathrm{C}$ (1 minuto), com primeiro gradiente de $25^{\circ} \mathrm{C} / \mathrm{min}$ até $160^{\circ} \mathrm{C}$, segundo gradiente de $50{ }^{\circ} \mathrm{C} / \mathrm{min}$ até $250^{\circ} \mathrm{C}$ (15 minutos), temperatura do detector em $300{ }^{\circ} \mathrm{C}$ e tempo da análise de 31 minutos.

Para a identificação dos agrotóxicos nas amostras essas foram injetadas no modo Single lon Monitoring (SIM) para possibilitar maior seletividade dos picos da amostra. A identificação (Tabela 1) ocorreu pela comparação do tempo de retenção absoluto dos padrões, presença dos íons selecionados e a razão entre os íons Qualificador e "Target", que devem ser equivalentes tanto nas amostras quanto nos padrões 
(FILLION, et al. 1995).

\begin{tabular}{|c|c|c|}
\hline & & \\
\hline Agrotoxicos & retençắo $(\mathrm{min})$ & Ions selecionados (SIM) \\
\hline $\begin{array}{l}\text { Clorotalanil } \\
\text { Heptacloro }\end{array}$ & $\begin{array}{r}12,42 \\
-13,3\end{array}$ & $266,264,268$ \\
\hline $\begin{array}{l}\text { Heptacloro } \\
\text { Aldrin }\end{array}$ & $\begin{array}{r}13,03 \\
13,4\end{array}$ & $272,372,274$ \\
\hline $\begin{array}{l}\text { Adrrin } \\
\text { Heptacloro epóxido }\end{array}$ & $\begin{array}{l}13,49 \\
14,02\end{array}$ & $\begin{array}{l}263,265,362 \\
353,355,386\end{array}$ \\
\hline Endosulfan alfa & 14,49 & $195,207,241,404$ \\
\hline Dieldrin & 14,82 & $263,277,378$ \\
\hline Endosulfan beta & 15,20 & $195,241,207,404$ \\
\hline Sulfato de E & 15,74 & $272,229,387,420$ \\
\hline
\end{tabular}

*Coluna HP5-MS (30 m x 0,25 mm di e 0,25 $\mu \mathrm{m}$ de filme), gás de arraste hélio, com vazão constante de $1,0 \mathrm{~mL} / \mathrm{min}$. Espectrômetro de massas operando em modo SIM-70 eV.

*De acordo com o estabelecido por FILLION et al. (1995).

\subsection{VALIDAÇÃO DO MÉTODO MODIFICADO DE LUKE et al. (1975)}

\subsubsection{Amostras, coleta e armazenamento}

Três amostras de $2 \mathrm{~kg}$ de repolho, tomate e batata in natura, sem lavar, escolhidas por representarem maior freqüência de consumo, foram coletadas no restaurante da UNICAMP. As amostras foram homogeneizadas em multiprocessador de alimentos (Walita), acondicionadas em frascos de vidro e armazenadas em congelador a $-18 \stackrel{\circ}{ } \mathrm{C}$ até 0 momento das análises (realizadas em duplicata).

\subsubsection{Recuperação do método}

Para os testes de recuperação, as amostras de repolho, tomate e batata foram fortificadas com $5 \mathrm{~mL}$ da mistura de soluções padrões preparadas em hexano com 5 níveis de fortificação $(0,02 ; 0,05 ; 0,10$; 0,15 e $0,2 \mathrm{mg} / \mathrm{kg}$ do fungicida Clorotalonil e dos inseticidas Aldrin, Dieldrin, Heptacloro, Heptacloro epóxido, endosulfan alfa, Endosulfan beta e Sulfato de Endosulfan). Os agrotóxicos foram identificados no cromatograma pela comparação dos tempos de retenção absolutos dos picos obtidos nas amostras fortificadas e não fortificadas com os tempos de retenção absolutos encontrados nos padrões analíticos submetidos às mesmas condições de análise. Calculou-se a porcentagem de recuperação pela diferença entre as áreas dos picos de cada analito com o mesmo tempo de retenção encontrado para amostras fortificada e não fortificada, dividida pela área correspondente ao pico do agrotóxico padrão considerado e 
multiplicado por cem. Todas as análises foram realizadas em duplicata.

\subsubsection{Limites de detecção e quantificação do método}

Os limites de detecção do método (LD) foram calculados conforme proposto por LONG e WINEFORDNER (1983) e LEITE (1998), segundo os quais tais limites são calculados a partir de 3 vezes o desvio do sinal da linha de base ou ruído do detector. Os limites de quantificação do método (LQ) foram calculados como 10 vezes o desvio do sinal produzido pela linha de base ou ruído, obtido a partir das curvas de calibração de cada um dos padrões (MILLER e MILLER, 1993).

\section{RESULTADOS E DISCUSSÃO}

A Tabela 2 mostra as concentrações, áreas e a equação da reta, obtidas nas curvas padrões para os agrotóxicos organoclorados.

TABELA 2 - CONCENTRAÇÕES, ÁREAS E EQUAÇÃO DA RETA DOS AGROTÓXICOS ESTUDADOS

\begin{tabular}{|c|c|c|c|}
\hline Agrotóxicos & $\begin{array}{c}\text { Concentração } \\
(\mathrm{mg} / \mathrm{L})\end{array}$ & Área & Equação da Reta \\
\hline \multirow{5}{*}{ Clorotalonil } & 0,025 & 3,873164 & \multirow{5}{*}{$\begin{array}{c}\mathrm{Y}=54,229 \mathrm{x}+2,5017 \\
\mathrm{R}^{2}=0,9996\end{array}$} \\
\hline & 0,0625 & 5,870000 & \\
\hline & 0,125 & 9,199877 & \\
\hline & 0,1875 & 12,83678 & \\
\hline & 0,25 & 15,97735 & \\
\hline \multirow{5}{*}{ Aldrin } & 0,025 & 3,273892 & \multirow{5}{*}{$\begin{array}{c}Y=45,964 x+2,1530 \\
R^{2}=0,9998\end{array}$} \\
\hline & 0,0625 & 4,985909 & \\
\hline & 0,125 & 7,994889 & \\
\hline & 0,1875 & 10,79959 & \\
\hline & 0,25 & 13,58746 & \\
\hline \multirow{5}{*}{ Dieldrin } & 0,025 & 3,863175 & \multirow{5}{*}{$\begin{array}{c}\mathrm{Y}=54,311 \mathrm{x}+2,4466 \\
\mathrm{R}^{2}=0,9997\end{array}$} \\
\hline & 0,0625 & 5,760000 & \\
\hline & 0,125 & 9,197868 & \\
\hline & 0,1875 & 12,73678 & \\
\hline & 0,25 & 15,97744 & \\
\hline \multirow{5}{*}{ Heptacloro } & 0,025 & 1,191101 & \multirow{5}{*}{$\begin{array}{c}Y=32,553 x+1,0179 \\
R^{2}=0,9990\end{array}$} \\
\hline & 0,0625 & 2,975129 & \\
\hline & 0,125 & 4,966266 & \\
\hline & 0,1875 & 7,231443 & \\
\hline & 0,25 & 9,152479 & \\
\hline \multirow{5}{*}{ Heptacloro epóxido } & 0,025 & 1,152122 & \multirow{5}{*}{$\begin{array}{c}Y=31,182 x+1,0099 \\
R^{2}=0,9991\end{array}$} \\
\hline & 0,0625 & 2,865228 & \\
\hline & 0,125 & 4,856255 & \\
\hline & 0,1875 & 6,986123 & \\
\hline & 0,25 & 8,756926 & \\
\hline \multirow{5}{*}{ Endosulfan alfa } & 0,025 & 1,035155 & \multirow{5}{*}{$\begin{array}{c}Y=31,036 x+0,2228 \\
R^{2}=0,9993\end{array}$} \\
\hline & 0,0625 & 2,135094 & \\
\hline & 0,125 & 4,13522 & \\
\hline & 0,1875 & 5,927261 & \\
\hline & 0,25 & 8,054673 & \\
\hline \multirow{5}{*}{ Endosulfan beta } & 0,025 & 0,961606 & \multirow{5}{*}{$\begin{array}{c}Y=24,345 x+0,2859 \\
R^{2}=0,9992\end{array}$} \\
\hline & 0,0625 & 1,706516 & \\
\hline & 0,125 & 3,350014 & \\
\hline & 0,1875 & 4,870219 & \\
\hline & 0,25 & 6,365534 & \\
\hline \multirow{5}{*}{ Sulfato de endosulfan } & 0,025 & 1,185034 & \multirow{5}{*}{$\begin{array}{c}Y=29,783 x+0,3566 \\
R^{2}=0,9994\end{array}$} \\
\hline & 0,0625 & 2,167266 & \\
\hline & 0,125 & 4,027215 & \\
\hline & 0,1875 & 5,895639 & \\
\hline & 0,25 & 7,866724 & \\
\hline
\end{tabular}




\subsection{LIMITES DE DETECÇÃO E QUANTIFICAÇÃO DO MÉTODO}

Na Tabela 3 estão apresentados os LD e LQ dos agrotóxicos organoclorados em repolho, tomate e batata. Para efeito de comparação foram também incluídos os limites máximos de resíduos (LMR) desses agrotóxicos autorizados para as diferentes hortaliças. A legislação brasileira estabelece LMR apenas para o Clorotalonil, em tomate e batata, e os limites de quantificação obtidos para esse composto nessas hortaliças foram inferiores aos respectivos limites. Esse resultado evidencia a aplicabilidade do método utilizado para o monitoramento de Clorotalonil nessas culturas.

Para os demais agrotóxicos não é possível estabelecer a mesma comparação em razão de uso não autorizado para as culturas estudadas. Entretanto, os valores mostraram-se baixos e inferiores aos encontrados por UNGARO et al. 1983; BARRETO et al. 1996; OLIVEIRA e TOLEDO, 1995. BARRETO et al. 1996, por exemplo, relataram limites de detecção de $0,01 \mathrm{mg} / \mathrm{kg}$ para os mesmos agrotóxicos organoclorados em alface, arroz, batata, cenoura, farinha de trigo, feijão, fubá e tomate.

\subsection{RECUPERAÇÃO DOS AGROTÓXICOS ORGANOCLORADOS}

No estudo de recuperação dos agrotóxicos, a coluna cromatográfica recheada com florisil p.r. foi eluída com 3 misturas de solventes percoladas em ordem crescente de polaridade. No eluente I (hexano: diclorometano 99:1 v:v) foram recuperados o Heptacloro e o Aldrin, no eluente II (hexano:diclorometano 80:20 v:v) o Heptacloro epóxido, o Endosulfan alfa e o Dieldrin, e no eluente III (hexano:diclorometano:acetonitrila 49:25:50,0:0,75 v:v) o Clorotalonil, o Endosulfan beta e o Sulfato de Endosulfan.

Os valores de recuperação encontrados nas diferentes hortaliças estão apresentados na Tabela 4. A recuperação média variou de 76,0 a $97,0 \%$ em repolho, de 90,0 a $103,0 \%$ em tomate e de 81,0 a $106,0 \%$ em batata. Esses resultados indicam que os valores de recuperação obtidos nas amostras analisadas, com exceção de Clorotalonil em repolho, enquadraram-se no limite de $80-110 \%$ recomendado pelo Pesticide Analytical Manual (FDA,1994) e no intervalo de $70-120 \%$, citado por PARKER (1991). Os coeficientes de variação (CV) oscilaram de 2,3 a $13,2 \%$, atendendo, portanto, ao valor limite de $16 \%$ aceito para análises de contaminantes em níveis de $\mathrm{mg} / \mathrm{kg}$ (HORWITZ, 1980). 


\section{TABELA 3 - LIMITES DE DETECÇÃO E QUANTIFICAÇÃO DOS AGROTÓXICOS ORGANOCLORADOS EM REPOLHO, TOMATE E BATATA}

\begin{tabular}{|c|c|c|c|}
\hline Agrotóxicos & LD (mg/kg) & LQ(mg/kg) & LMR $(\mathrm{mg} / \mathrm{kg})$ \\
\hline \multicolumn{4}{|l|}{ Repolho } \\
\hline Clorotalonil & 0,0006 & 0,002 & na \\
\hline Aldrin & 0,0006 & 0,002 & na \\
\hline Dieldrin & 0,0008 & 0,003 & na \\
\hline Heptacloro & 0,0006 & 0,002 & na \\
\hline Heptacloro epóxido & 0,0007 & 0,003 & na \\
\hline Endosulfan alfa & 0,0008 & 0,003 & na \\
\hline Endosulfan beta & 0,0009 & 0,003 & na \\
\hline Sulfato de Endosulfan & 0,0016 & 0,005 & na \\
\hline \multicolumn{4}{|l|}{ Tomate } \\
\hline Clorotalonil & 0,0006 & 0,002 & 1,00 \\
\hline Aldrin & 0,0006 & 0,002 & na \\
\hline Dieldrin & 0,0008 & 0,003 & na \\
\hline Heptacloro & 0,0006 & 0,002 & na \\
\hline Heptacloro epóxido & 0,0007 & 0,003 & na \\
\hline Endosulfan alfa & 0,0008 & 0,003 & na \\
\hline Endosulfan beta & 0,0009 & 0,003 & na \\
\hline Sulfato de Endosulfan & 0,0016 & 0,005 & na \\
\hline \multicolumn{4}{|l|}{ Batata } \\
\hline Clorotalonil & 0,0006 & 0,002 & 0,10 \\
\hline Aldrin & 0,0006 & 0,002 & na \\
\hline Dieldrin & 0,0008 & 0,003 & na \\
\hline Heptacloro & 0,0006 & 0,002 & na \\
\hline Heptacloro epóxido & 0,0007 & 0,003 & na \\
\hline Endosulfan alfa & 0,0008 & 0,003 & na \\
\hline Endosulfan beta & 0,0009 & 0,003 & na \\
\hline Sulfato de Endosulfan & 0,0016 & 0,005 & na \\
\hline
\end{tabular}

LD = Limites de detecção.

$\mathrm{LQ}=$ Limites de quantificação.

LMR = Limite Máximo de Resíduo, Legislação Brasileira (1999).

na $=$ não autorizado.

\subsection{DETERMINAÇÃO DE AGROTÓXICOS ORGANOCLORADOS EM HORTALIÇAS}

As Tabelas 5 e 6 mostram os níveis de resíduos dos agrotóxicos estudados em hortaliças servidas nos restaurantes da UNICAMP e coletadas na CEASA de Campinas. Em todas as amostras $(n=151)$, os níveis dos agrotóxicos mostraram-se inferiores aos respectivos limites 
de detecção. Resultados semelhantes foram obtidos no monitoramento de resíduos de agrotóxicos organoclorados conduzido por BARRETO et al. (1996), em 242 amostras de cereais, legumes e vegetais comercializados no Estado de São Paulo, no ano de 1994. Com exceção de uma amostra de tomate, que continha $0,01 \mathrm{mg} / \mathrm{kg}$ de Endosulfan, os demais alimentos pesquisados apresentaram níveis de agrotóxicos abaixo do limite de detecção analítica dos organoclorados analisados $(0,01 \mathrm{mg} /$ $\mathrm{kg}$ ). Tais resultados evidenciam o uso inadequado do Endosulfan em tomate e o cumprimento das boas práticas agrícolas e da legislação em vigor para as demais culturas.

\section{TABELA 4- RECUPERAÇÃODOSAGROTÓXICOSORGANOCLORADOS EM REPOLHO, TOMATE E BATATA}

\begin{tabular}{|c|c|c|c|}
\hline Agrotóxicos & Recuperação* (\%) & $\begin{array}{c}\text { Estimativa do } \\
\text { Desvio Padrão }( \pm)\end{array}$ & $\begin{array}{c}\text { Coeficiente de Variação } \\
(\%)\end{array}$ \\
\hline \multicolumn{4}{|l|}{ Repolho } \\
\hline Clorotalonil & 76,0 & 10,0 & 13,2 \\
\hline Aldrin & 82,0 & 10,0 & 12,2 \\
\hline Dieldrin & 95,0 & 11,0 & 11,6 \\
\hline Heptacloro & 83,0 & 8,0 & 9,6 \\
\hline Heptacloro epóxido & 81,0 & 8,0 & 9,9 \\
\hline Endosulfan alfa & 88,0 & 9,0 & 10,2 \\
\hline Endosulfan beta & 94,0 & 10,0 & 10,6 \\
\hline Sulfato de Endosulfan & 97,0 & 11,0 & 11,3 \\
\hline \multicolumn{4}{|l|}{ Tomate } \\
\hline Clorotalonil & 93,0 & 12,0 & 12,9 \\
\hline Aldrin & 90,0 & 4,0 & 4,4 \\
\hline Dieldrin & 99,0 & 3,0 & 3,0 \\
\hline Heptacloro & 90,0 & 5,0 & 5,6 \\
\hline Heptacloro epóxido & 95,0 & 12,0 & 12,6 \\
\hline Endosulfan alfa & 98,0 & 10,0 & 10,2 \\
\hline Endosulfan beta & 103,0 & 7,0 & 6,8 \\
\hline Sulfato de Endosulfan & 103,0 & 9,0 & 8,7 \\
\hline \multicolumn{4}{|l|}{ Batata } \\
\hline Clorotalonil & 85,0 & 9,0 & 10,6 \\
\hline Aldrin & 81,0 & 9,0 & 11,1 \\
\hline Dieldrin & 99,0 & 11,0 & 11,1 \\
\hline Heptacloro & 86,0 & 2,0 & 2,3 \\
\hline Heptacloro epóxido & 87,0 & 9,0 & 10,3 \\
\hline Endosulfan alfa & 97,0 & 11,0 & 11,3 \\
\hline Endosulfan beta & 100,0 & 9,0 & 9,0 \\
\hline Sulfato de Endosulfan & 106,0 & 12,0 & 11,3 \\
\hline
\end{tabular}

* Médias das recuperações correspondentes aos níveis de fortificação: 0,02; 0,05; 0,$10 ; 0,15$ e $0,20 \mathrm{mg} / \mathrm{kg}$ (2 repetições). 


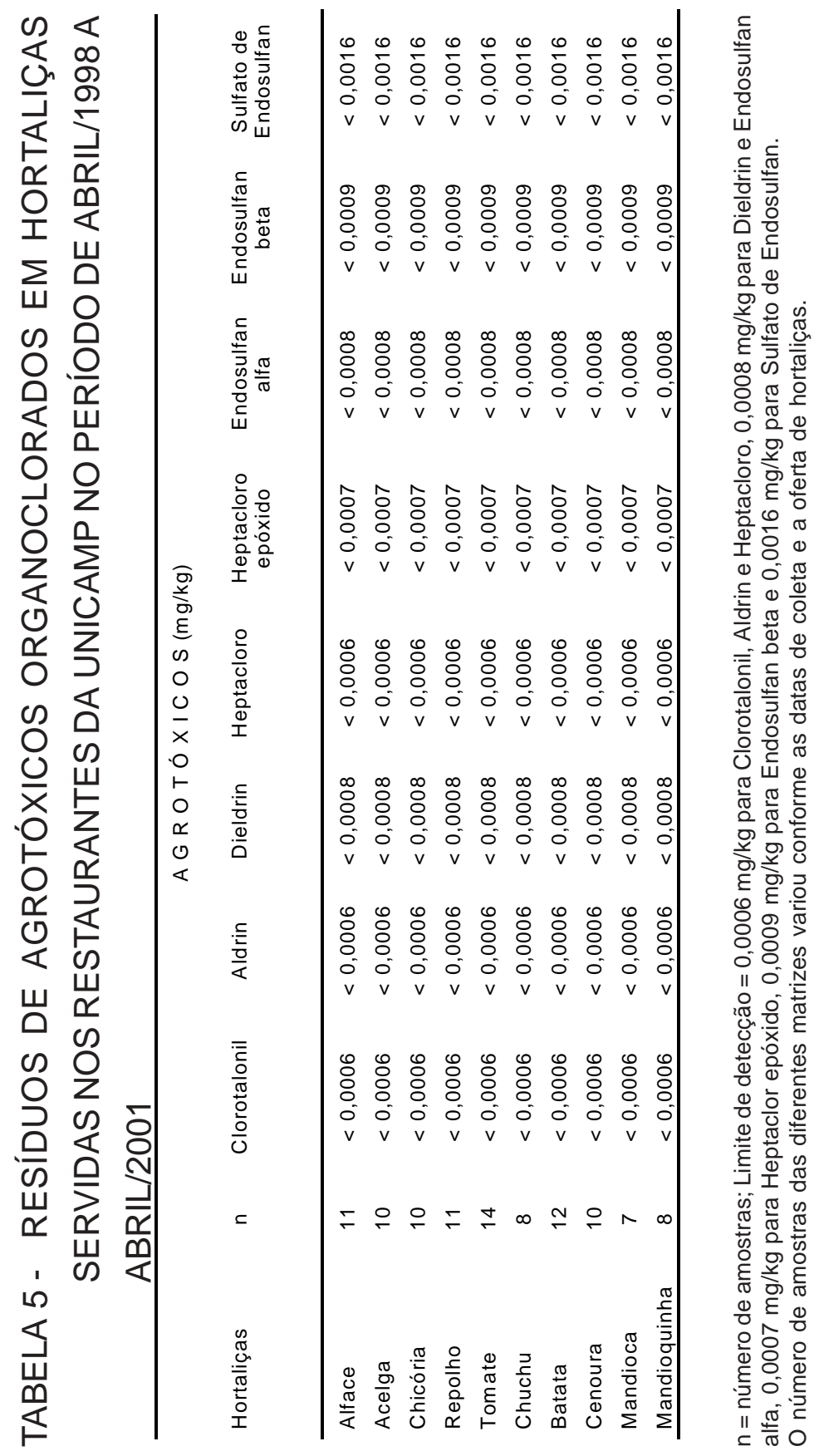




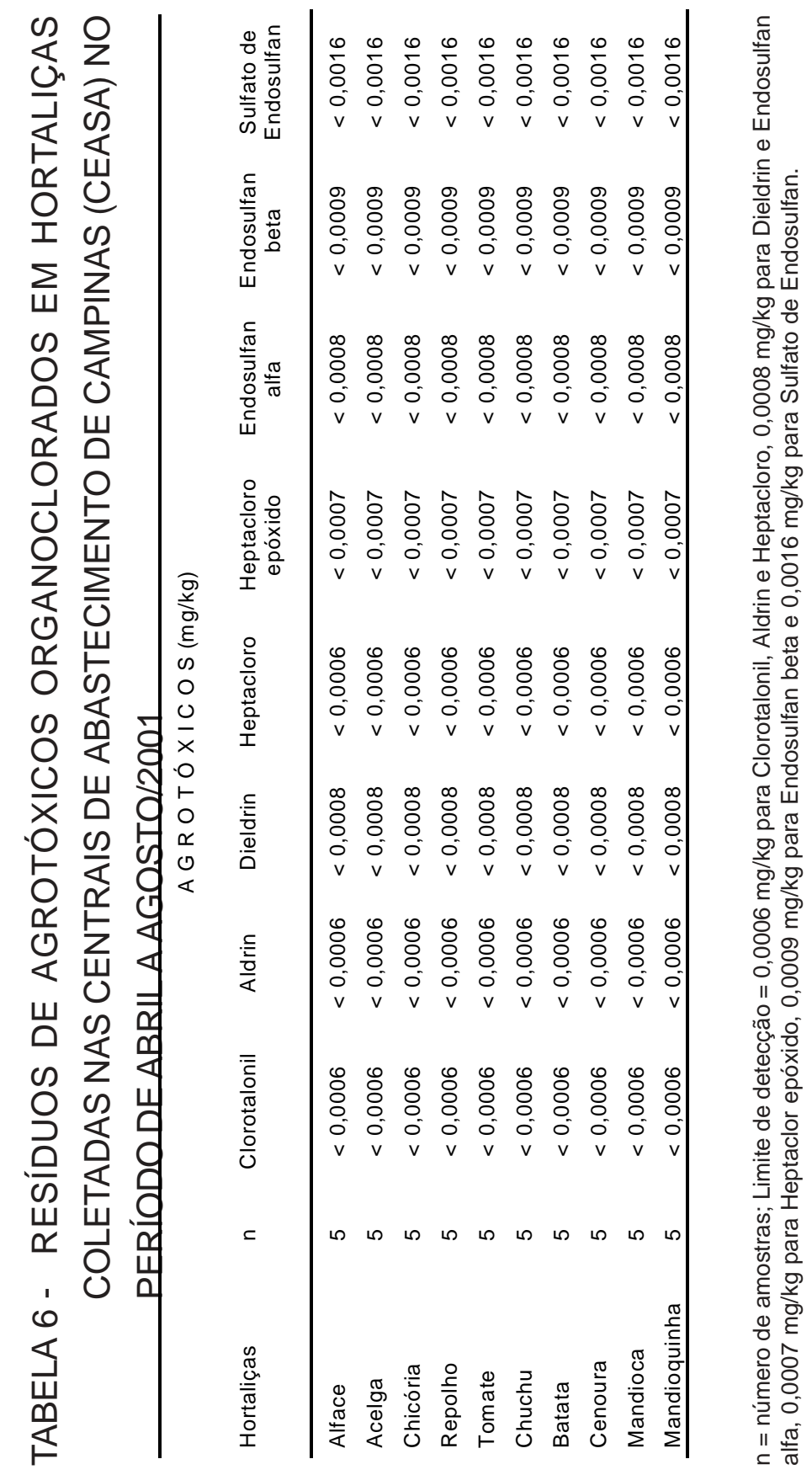


Nas Figuras 1, 2 e 3 são apresentados os cromatogramas referentes aos padrões e amostra de repolho para os três diferentes eluentes.

FIGURA 1 - CROMATOGRAMAS POR CG-DCE CORRESPONDENTES A: A) MISTURA DE PADRÕES $0,1 \mathrm{mg} / \mathrm{L}$ DE CLOROTALONIL (1), HEPTACLOR (2), ALDRIN (3), HEPTACLOR HEPOXI (4), ENDOSULFAN ALFA (5) E DIELDRIN (6), ENDOSULFAN BETA (7), SULFATO DE ENDOSULFAN (8), B) AMOSTRA DE REPOLHO FORTIFICADA COM $5 \mathrm{~mL}$ DA MISTURA DE PADRÕES DE CONCENTRAÇÃO $1,0-1,3 \mathrm{mg} / \mathrm{L}$. HEPTACLOR (2), ALDRIN (3), C) AMOSTRA DE REPOLHO NÃO FORTIFICADA. ELUENTE I (HEXANO:DICLOROMETANO 99:1 V:V) E I-PICO IDENTIFICADO COMO INTERFERENTE NA MATRIZ DE REPOLHO
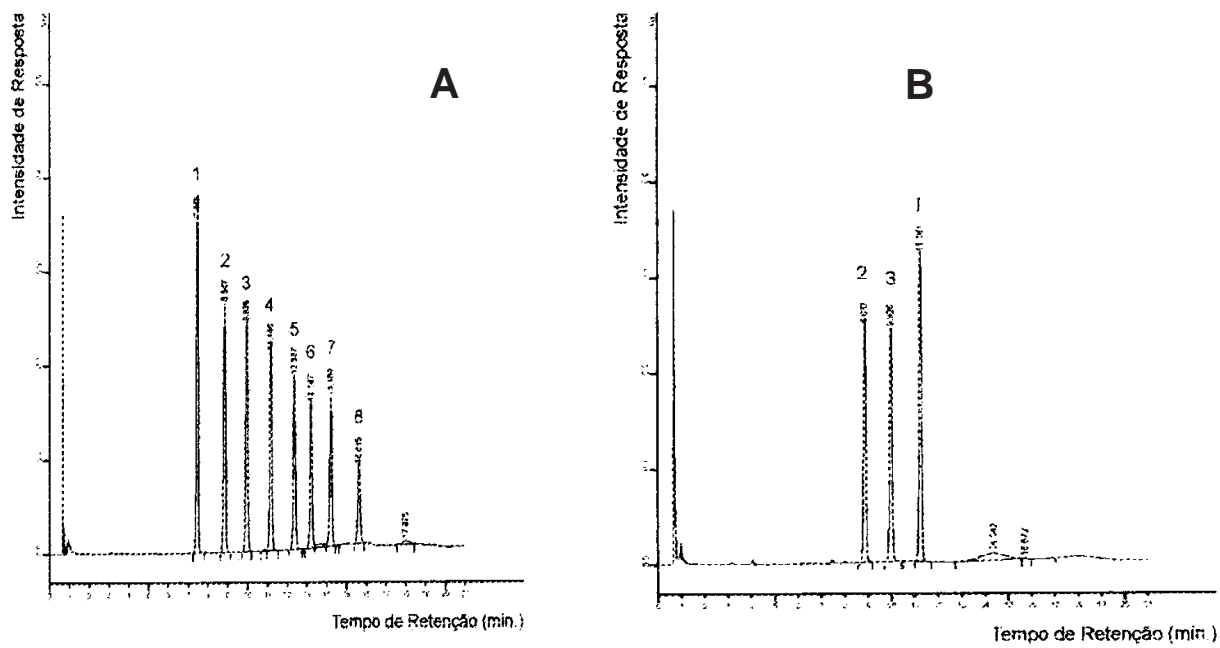

FIGURA 2 -

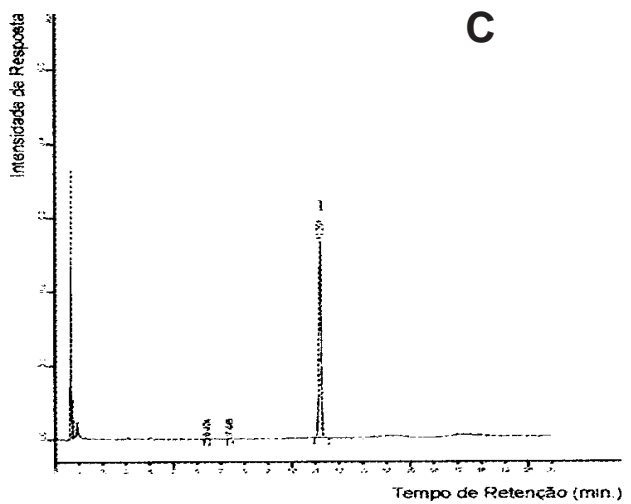

S A: D) AMOSTRA 
DE REPOLHO NÃO FORTIFICADA, E) AMOSTRA DE REPOLHO FORTIFICADA COM $5 \mathrm{~mL}$ DA MISTURA DE PADRÕES DE CONCENTRAÇÃO 1,0 - 1,3 mg/L. HEPTACLORO EPÓXIDO (4), ENDOSULFAN ALFA (5) E DIELDRIN (6). ELUENTE II (HEXANO:DICLOROMETANO 80:20 V:V)
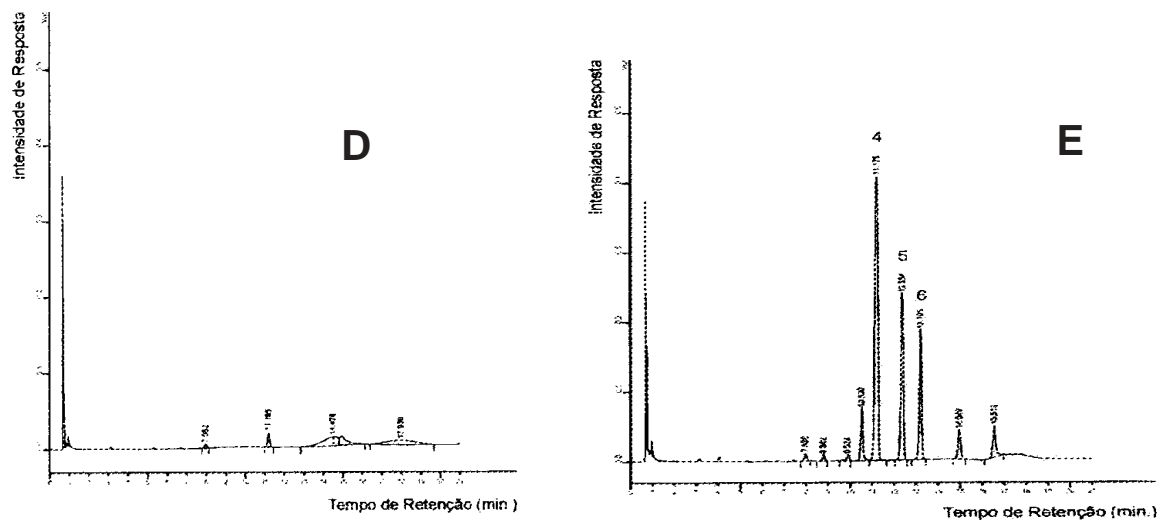

FIGURA 3 - CROMATOGRAMAS POR CG-DCE CORRESPONDENTES A: F) AMOSTRA DE REPOLHO NÃO FORTIFICADA E G) AMOSTRA DE REPOLHO FORTIFICADA COM 5 mL DA MISTURA DE PADRÕES, CONCENTRAÇÃO 1,0 - 1,3 mg/L. CLOROTALONIL (1), ENDOSULFAN BETA (7) E SULFATO DE ENDUSULFAN (8). ELUENTE III (HEXANO:DICLOROMETANO:ACETONITRILA 49,25:50,0:0,75 V/V)
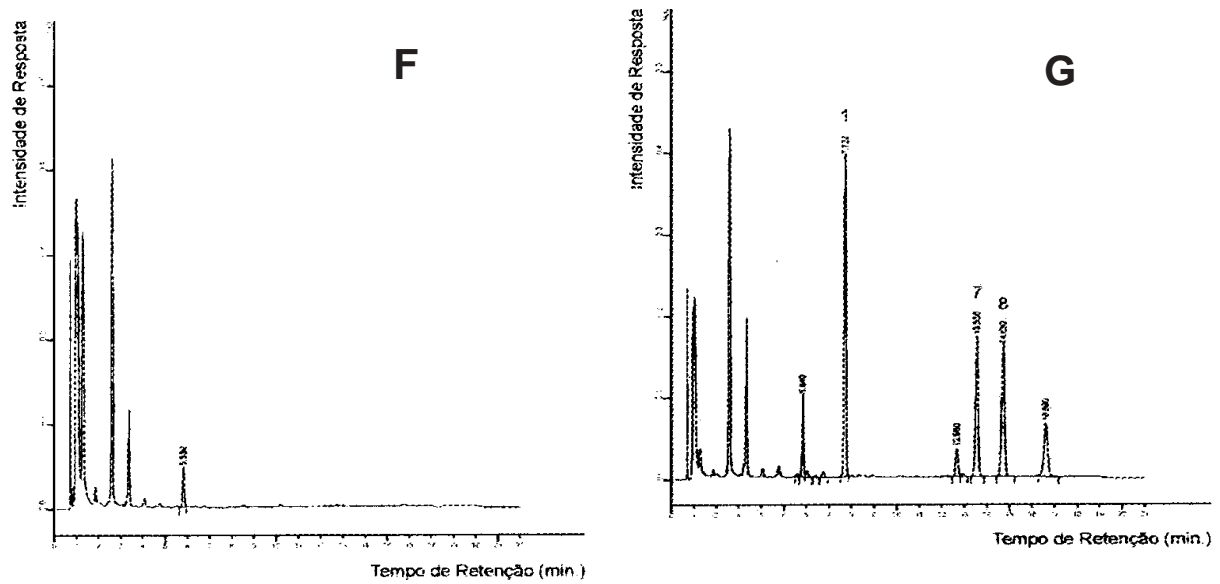
Informações adicionais sobre a origem das hortaliças analisadas foram obtidas com o responsável pelo seu suprimento ao restaurante da UNICAMP. Verificou-se que as hortaliças adquiridas pela Universidade são provenientes de três agricultores estabelecidos nos municípios de Salto, Guapiara e Sousas (pertencentes ao Distrito de Campinas), os quais não utilizam agrotóxicos em suas lavouras. Essa prática foi substituída pela rotação de culturas, sendo tomados cuidados especiais na limpeza das hortas e na aplicação de adubo orgânico, quando necessário.

Não foram detectados resíduos de Clorotalonil, Aldrin, Dieldrin, Heptacloro, Heptacloro epóxido, Endosulfan alfa, Endosulfan beta e sufato de Endosulfan nas amostras analisadas de alface, acelga, chicória, repolho, tomate, chuchu, batata, cenoura, mandioca e mandioquinha comercializadas nas Centrais de Abastecimento de Campinas (CEASA) e coletadas mensalmente por 5 meses consecutivos. Apenas em uma amostra de cenoura e uma de chuchu obteve-se para o eluente III cromatogramas com perfil diferente, evidenciando um pico no tempo de retenção do Sulfato de Endosulfan. Entretanto, essa suspeita não foi confirmada analiticamente.

Mesmo depois de 6 anos do monitoramento conduzido por BARRETO et al. (1996), os resultados obtidos no presente estudo indicam que a legislação vigente ainda está sendo cumprida e que as boas práticas agrícolas continuam sendo adotadas na região estudada.

\section{CONCLUSÃO}

O método multirresíduo de Luke apresentou recuperações médias na faixa de $70 \%$ a $120 \%$, valores aceitos internacionalmente e precisão $\mathrm{CV}<16 \%$. Os limites de detecção e quantificação mostraram-se adequados aos níveis dos agrotóxicos avaliados. A linearidade de resposta também foi considerada satisfatória para a faixa de concentração estudada.

Os resultados obtidos permitem afirmar que as hortaliças servidas nos restaurantes Universitários da UNICAMP, no período de abril/1998 a abril/2001, e as comercializadas nas Centrais de Abastecimento de Campinas (CEASA), de abril a agosto de 2001, atenderam à legislação brasileira para resíduos de agrotóxicos organoclorados e que boas práticas agrícolas foram seguidas com relação ao uso dos agrotóxicos analisados. 


\begin{abstract}
DETERMINATION OF ORGANOCHLORINE PESTICIDES RESIDUES ON VEGETABLES

Residues of Chlorotalonil, Aldrin, Dieldrin, Heptachlor, Heptachlor epoxide, alpha and beta Endosulfan and Endosulfan sulfate were analysed in the following vegetables: lettuce, swiss chad, chicory, cabbage, tomato, chayote, potato, carrot, cassava and cassava specy. The samples were collected at the restaurant of the University of Campinas, from April/1998 to April/2001 at the "Centrais de Abastecimento de Campinas (CEASA)", from April to August/2001. The organochlorine pesticide residues were determined by gas chromatography with an electron capture detector (ECD- $\left.{ }^{63} \mathrm{Ni}\right)$. The detection limits of Chlorotalonil, Aldrin, Dieldrin, Heptachlor, Heptachlor epoxide, alpha and beta Endosulfan and Endosulfan sulfate were 0.0006, 0.0006, 0.0008, 0.0006, $0.0007,0.0008,0.0009$ and $0.0016 \mathrm{mg} / \mathrm{kg}$ respectively, and their quantification limits were $0.002,0.002,0.003,0.002,0.003,0.003,0.003$ and $0.005 \mathrm{mg} / \mathrm{kg}$. The average recovery of the pesticides varied between 76.0 and $97.0 \%$ for cabbage, 90,0 to $103.0 \%$ for tomato and 81.0 to $106.0 \%$ for potato. The samples analysed $(n=151)$ showed pesticide levels below the detection limits of the validated method.

KEY-WORDS: RESIDUES; PESTICIDES-ORGACHLORINE; VEGETABLES.
\end{abstract}

\title{
REFERÊNCIAS
}

1 ANVISA. Agência Nacional de Vigilância Sanitária. C18Clorotalonil. Disponível em: <http://anvisa.gov.br/alimentos/ tox/mono/c18.htm>. Acesso em 17 jun. 2002a.

2 ANVISA. Agência Nacional de Vigilância Sanitária. E02Endosulfan. Disponível em: <http://anvisa.gov.br/alimentos/ tox/mono/e 02.htm>. Acesso em 17 jun. 2002b.

3 BARRETO. H. C. C.; INOMATA, O. N.; LEMES, V. R. R.; KUSSUMI, T. A.; SCORSAFAVA, M. A.; ROCHA, S. O. R. Monitoramento de resíduos de pesticidas em alimentos comercializados no estado de São Paulo em 1994. Pesticidas: Revista Técnico Científica, Curitiba, v. 6, p. 112, jan./dez., 1996.

4 BRASIL. Ministério da Agricultura. Portaria n. 329 de 02 de setembro de 1985. Proíbe em todo o território nacional a comercialização, o uso e a distribuição de produtos agrotóxicos organoclorados destinados á agropecuária. Diário Oficial da República Federativa do Brasil, Brasília, 
3 set. 1985. Seção I, p. 12941.

5 BRASIL. Ministério da Saúde. Relação de susbstâncias para uso domissanitário: Portarias do Ministério da Saúde. São Paulo: International Life Sciences Institute (ILSI), 1995. Disponível em: <http://anvs1.saude.gov.br/toxicologia/ toxicologia/html\%20monografias>. Acesso em 03 jan. 1999.

6 FDA. Food and Drug Administration. Pesticide analytical manual: multiresidue methods. $3^{\text {rd }}$ ed. Washington, 1994. v. 1 , p. 103-302b.

7 FILLION, J.; HINDLE, R.; LACROIX, M.; SELWYN, J. Multiresidue determination of pesticides in fruit and vegetables by gas chromatography-mass-seletive detection and liquid chromatography with fluorescence detection. Journal of the Association of Official Analytical Chemists International, Arlington, v. 78, n. 5, p. 1252-1266, p. 31-34, Set./Oct. 1995.

8 HORWITZ, W.; KAMPS, L. R.; BOYER, K. W. Quality assurance in the analysis of food for trace constiuents. Journal of the Association of Official Analytical Chemists, Arlington, v. 63, n. 6, p. 1344-1345, Nov., 1980.

9 LEITE, F. Sensibilidade, seletividade e limites de determinação e detecção. In: LEITE, F. Validação em análise química. 3.ed., Campinas: Átomo, 1998. Cap. 13, p.67-69.

10 LONG, G. L.; WINEFORDNER, J. D. Limit of detection: a closer look at the IUPAC definition. Analytical Chemistry, Washington, v. 55, p. 712-724A, 1983.

11 LUKE, M. A.; FROBERG, J. E.; MASUMOTO, H. T. Extraction and cleanup of organochlorine, organophosphate, organonitrogen and hidrocarbon pesticides in produce for determination by gas-liquid chromatography. Journal of the Association of Official Analytical Chemists, Washington, v. 58, n. 5, p. 1020-1026, 1975. 
12 MILLS, P. A.; BONG, B.A.; KAMPS, L.R.; BURKE, J.A. Elution solvent system for florisil column in organochlorine pesticide residue analyses. Journal of the Association of Official Analytical Chemists, Washington, v. 55, n. 1, p. 39-43, 1972.

13 MILLER, J. C.; MILLER, J. N. Error in instrumental analysis; regression and correlation. In: MILLER, J.C.; MILLER, J.N. Statistic for analytical chemistry. $3^{\text {rd }}$ ed. Chichester: Ellis Horwood, 1993. Chapter 5, p. 101-141.

14 OLIVEIRA, J.J.V.; TOLEDO, M.C.F. Resíduos de agrotóxicos em morangos. Pesticidas: Revista de Ecotoxicologia e Meio Ambiente, Curitiba, v. 5, p. 95-109, jan./dez., 1995.

15 PARKER, G.A. Validation of methods used in the Florida departament of agriculture and consumer services chemical residue laboratory. Journal of Association of Official Analytical Chemists, Arlington, v. 74, n. 5, p. 868-871, Sept./ Oct., 1991.

16 UNGARO, M.T.S.; PIGATI, P.; GUINDANI, C.M.A.; FERREIRA, M.S.; GEBARA, A.B.; ISHIZAKI, T. Resíduos de inseticidas clorados e fosforados em frutas e hortaliças (II). Biológico, São Paulo, v. 49, n. 1, p. 1-8, jan., 1983.

17 UNGARO, M.T.S.; GUINDANI, C.M.A.; FERREIRA, M.S.; BAGDONAS, M. Resíduos de inseticidas clorados e fosforados em frutas e hortaliças (II). Biológico, São Paulo, v. 52, n. 7/12, p. 51-56, jul./dez., 1987.

18 YOKOMIZO, Y.; LARA, W.H.; PUGA, F.; BATISTA, G. C.; CARVALHO, P. R. N.; BARRETO, H. H. C.; MALTONE, C. A.; DOEGE, M. Resíduos de pesticidas em alimentos. Campinas: ITAL, 1984. 227 p.

19 ZANDONÁ, M.S.; ZAPPIA, V.R.S. Resíduos de agrotóxicos em alimentos: Resultado de cinco anos de monitoramento realizado pela secretaria de saúde do Paraná. Pesticidas: Revista Técnico Científica, v. 3, n. 3, jan./dez., p. 49-95, 1993. 


\section{Agradecimentos}

À FAPESP, pela concessão de bolsa de doutorado no país, Proc.97/09620-7, e reserva técnica correspondente, Proc.97/11887-1. Ao Instituto de Tecnologia de Alimentos (ITAL) de Campinas, pelo apoio e suporte técnico. 